Pesq. Vet. Bras. 35(9):788-794, setembro 2015

DOI: $10.1590 / \mathrm{S} 0100-736 \mathrm{X} 2015000900004$

\title{
Parasitos de aves e mamíferos silvestres em cativeiro no estado de Pernambuco ${ }^{1}$
}

\author{
Pauline Marie de Souza Santos ${ }^{2}$, Silvia Gabriela Nunes da Silva², Cristina Farias da \\ Fonseca $^{3}$ e Jaqueline Bianque de Oliveira ${ }^{2 *}$
}

\begin{abstract}
Santos P.M.S., Silva S.G.N., Fonseca C.F. \& Oliveira J.B. 2015. [Parasites of birds and mammals in captivity in Pernambuco state, Brazil.] Parasitos de aves e mamíferos em cativeiro no estado de Pernambuco. Pesquisa Veterinária Brasileira 35(9):788-794. Laboratório de Parasitologia, Departamento de Biologia, Universidade Federal Rural de Pernambuco, Rua Dom Manoel de Medeiros s/n, Recife, PE 52171-900, Brazil. E-mail: bianque01@yahoo.com.br

Wild animals are host to a variety of parasites that may interfere in their ex situ conservation. The objective of this study was to identify gastrointestinal parasites (GP) and ectoparasites of animals of Centro de Triagem de Animais Silvestres (CETAS) of Instituto Brasileiro de Meio Ambiente e Recursos Renováveis (IBAMA) in Recife/Pernambuco, Brazil, and to determine the aspects of captivity management that may be related to the parasites identified. We collected ectoparasites and fecal samples from 223 birds and mammals, which were processed by direct smear, flotation and sedimentation methods. Overall, helminthes and/ or protozoa were detected in 91 (40.8\%) fecal samples of $64(70.3 \%)$ birds and 27 (29.7\%) mammals. Capillaria sp., Ascaridida and Spirurida eggs and Eimeria sp. oocysts were found in fecal samples of birds, while eggs of Trichuris trichiura, Strongyloides sp., Toxocara canis, Ancylostoma sp., Strongylida and Coccidia oocysts were detected in fecal samples of mammals. Ectoparasites identified in birds were Colpocephalum turbinatum, Kurodaia (Kurodaia) fulvofasciata, Halipeurus sp., Naubates sp., Saemundssonia sp., Austromenopon sp., Paragoniocotes sp., Brueelia sp., Myrsidea sp. and Pseudolynchia sp. In mammals, the ectoparasites identified were Rhipicephalus sanguineus, Amblyomma varium, A. calcaratum, A. nodosum, Ornithodoros talaje and Ctenocephalides felis felis. A. calcaratum e $O$. talaje are presented for the first time in Pernambuco state and T. tetradactyla is reported as a new host of $O$. talaje. No animal presented clinical signs due to parasitic infection/infestation. Zoonotic parasites like T. trichiura, Strongyloides sp., T. canis and Ancylostoma sp. were identified in non-human primates and carnivores. Poor structural and sanitary conditions of CETAS-PE are related to the parasites identified in this study and should be taken into account for the adoption of appropriate control measures. The results of this study will contribute significantly to the conservation of wildlife in CETAS-PE and health of the professionals responsible for maintaining these animals.
\end{abstract}

INDEX TERMS: Gastrointestinal parasites, ectoparasites, control, biodiversity, ex situ conservation.

RESUMO.- Os animais silvestres são hospedeiros de uma grande variedade de parasitos que podem interferir em sua conservação ex situ. 0 objetivo deste estudo foi identificar

\footnotetext{
${ }^{1}$ Recebido em 23 de março de 2015.

Aceito para publicação em 10 de setembro de 2015.

${ }^{2}$ Laboratório de Parasitologia (LAPAR), Departamento de Biologia, Universidade Federal Rural de Pernambuco (UFRPE), Rua Dom Manoel de Medeiros s/n, Recife, PE 52171-900, Brasil. *Autor para correspondência: bianque01@yahoo.com.br

${ }^{3}$ Centro de Triagem de Animais Silvestres (CETAS), Instituto Brasileiro de Meio Ambiente e Recursos Naturais (IBAMA), Avenida 17 de agosto 1057, Recife, PE 52060-590.
}

os parasitos gastrointestinais (PGI) e ectoparasitos dos animais do Centro de Triagem de Animais Silvestres (CETAS) do Instituto Brasileiro de Meio Ambiente e Recursos Naturais Renováveis (IBAMA) de Recife, Pernambuco, além de determinar os aspectos do manejo em cativeiro que possam estar relacionados com os parasitos identificados. Foram coletados ectoparasitos e amostras fecais de 223 aves e mamíferos, as quais foram processadas pelos métodos: microscopia direta, flutuação e sedimentação. Helmintos e/ ou protozoários foram detectados em $91(40,8 \%)$ amostras fecais, sendo 64 (70,3\%) de aves e 27 (29,7\%) de mamíferos. Ovos de Capillaria sp., Ascaridida, Spirurida e oocistos 
de Eimeria sp. foram detectados nas amostras fecais das aves, enquanto ovos de Trichuris trichiura, Strongyloides sp., Toxocara canis, Ancylostoma sp., Strongylida e oocistos de Coccídios foram encontrados nas amostras fecais de mamíferos. Os ectoparasitos identificados em aves foram $\mathrm{Col}$ pocephalum turbinatum, Kurodaia (Kurodaia) fulvofasciata, Halipeurus sp., Naubates sp., Saemundssonia sp., Austromenopon sp., Paragoniocotes sp., Brueelia sp., Myrsidea sp. and Pseudolynchia sp., enquanto em mamíferos os ectoparasitos identificados foram Rhipicephalus sanguineus, Amblyomma varium, A. calcaratum, A. nodosum, Ornithodoros talaje e Ctenocephalides felis felis. A. calcaratum e 0 . talaje são registrados pela primeira vez em Pernambuco e T. tetradactyla é apresentado como novo hospedeiro de 0 . talaje. Nenhum dos animais estudados apresentou sinais clínicos em decorrência da infecção/infestação parasitária. Parasitos com potencial zoonótico como T. trichiura, Strongyloides sp., T. canis e Ancylostoma sp. foram identificados em primatas não humanos e carnívoros. Precárias condições estruturais e sanitárias do CETAS-PE estão relacionadas com os parasitos identificados neste estudo e devem ser levadas em consideração para a adoção de medidas adequadas de controle. Os resultados deste estudo contribuirão de maneira significativa para a conservação de animais selvagens no CETAS-PE e para a saúde dos profissionais responsáveis pela manutenção destes animais.

TERMOS DE INDEXAÇÃO: Parasitos gastrointestinais, ectoparasitos, controle, biodiversidade, conservação ex situ.

\section{INTRODUÇÃO}

Estima-se que cerca de 38 milhões de animais sejam retirados anualmente da natureza por traficantes (Renctas 2001) e que esta atividade ilegal movimente cifras em torno de US\$ 10 bilhões/ano em todo o mundo (Pagano et al. 2009). Recife e Petrolina (PE), Itabaiana (SE), Salvador, Feira de Santana e Paulo Afonso (BA), Crato (CE) e Picos (PI) são as principais cidades envolvidas no tráfico de animais silvestres na região Nordeste do Brasil (Pagano et al. 2009). Os Centros de Triagem de Animais Silvestres (CETAS) do Instituto Brasileiro de Meio Ambiente e Recursos Naturais Renováveis (IBAMA), foram criados com o objetivo principal de receber e manter animais silvestres (oriundos de apreensões realizadas pelos órgãos ambientais, entrega voluntária e resgate) até que estes possam ser transferidos para um zoológico ou criadouro cadastrado, ou então quando o processo de reabilitação possibilite a reintrodução do animal na natureza (Brasil 2003).

Os animais silvestres são hospedeiros de uma grande variedade de parasitos, que podem atuar como oportunistas ou como agentes primários de doença (Freitas et al. 2001 e 2002a, Catão-Dias 2003, Godoy \& Cubas 2011, Oliveira et al. 2011, Santos et al. 2011). Segundo Daszak et al. (2000), os parasitos patogênicos podem representar uma ameaça para os programas de manejo e recuperação de populações animais, o que assume particular importância para espécies ameaçadas. Neste sentido, as infecções parasitárias são uma das principais doenças que acometem animais silvestres em cativeiro, estando a morbidade e mortalidade das infecções e/ou infestações parasitárias dependente de variáveis tais como a espécie de parasito e a carga parasitária, estado nutricional, imunocompetência e condições fisiológicas do hospedeiro (Catão-Dias 2003, Godoy \& Cubas 2011). Por isso, a identificação da fauna parasitária é exigida em protocolos de reintrodução (UICN 1998, Felasa 1999) e na rotina clínica de animais silvestres (Freitas et al. 2001, 2002a, Deem et al. 2008, Godoy \& Cubas 2011, Oliveira et al. 2011, Santos et al. 2011). Deve-se, também, considerar o potencial zoonótico de alguns parasitos (Daszak et al. 2000, Freitas et al. 2001, Sibaja-Morales et al. 2009). Desta maneira, o objetivo deste estudo foi identificar os parasitos gastrointestinais (PGI) e ectoparasitos dos animais silvestres abrigados no Centro de Triagem de Animais Silvestres (CETAS) do Instituto Brasileiro de Meio Ambiente e Recursos Naturais Renováveis (IBAMA) de Recife, Pernambuco, além de determinar os aspectos do manejo em cativeiro que possam estar relacionados com os parasitos identificados.

\section{MATERIAL E MÉTODOS}

O estudo foi realizado, no período de setembro de 2012 a fevereiro de 2013, no CETAS do Recife, localizado no bairro de Casa Forte, na cidade do Recife, Pernambuco. Fizeram parte do estudo tanto os animais de ingresso recente quanto aqueles que já estavam abrigados no CETAS há algum tempo. Os animais eram oriundos de apreensões e resgates realizados por órgãos ambientais, assim como por entrega voluntária por parte da população. Para a maioria dos animais analisados não havia registro de sua origem.

As amostras fecais foram coletadas quinzenalmente, mas cada animal só foi amostrado uma única vez e, para nenhum deles, havia registro de tratamento antiparasitário prévio. As amostras foram acondicionadas em recipientes plásticos identificados e transportadas em caixas isotérmicas com gelo para o Laboratório de Parasitologia (LAPAR) do Departamento de Biologia da Universidade Federal Rural de Pernambuco (UFRPE). As amostras foram mantidas sob a refrigeração até seu processamento. Para o diagnóstico de parasitos gastrointestinais (PGI), as amostras foram processadas pelos métodos: microscopia direta, flutuação de Sheather modificado (com solução hipersaturada de açúcar) (Sibaja-Morales et al. 2009, Santos et al. 2011) e de sedimentação espontânea (Freitas et al. 2001, 2002a). A identificação dos oocistos e ovos de PGI seguiu a descrição morfológica de Zajac \& Conboy (2006), Urquhart et al. (2008) e Kouassi et al. (2015).

Os animais foram sistematicamente inspecionados (com o auxílio de uma lupa) e os ectoparasitos foram removidos e acondicionados em frascos contendo etanol $70^{\circ} \mathrm{GL}$ para posterior identificação no LAPAR.

Os piolhos foram clarificados em uma bateria de fenol-xilol (Freitas et al. 2002b, Melo et al. 2012) e montados entre lâmina e lamínula com resina sintética permanente (Entellan®). Para identificação, foram utilizados a chave de Price et al. (2003) e artigos científicos especializados. Os carrapatos foram identificados em microscópio estereoscópico, utilizando as chaves de Aragão \& Fonseca (1961), Barros-Battesti et al. (2006) e Martins et al. (2010).

As pulgas foram clarificadas com hidróxido de potássio $(\mathrm{KOH})$ a $10 \%$ e montadas entre lâmina e lamínula com resina sintética permanente (Entellan®) (Freitas et al. 2002b, Oliveira et al. 2011). A identificação seguiu os padrões morfológicos descritos por Linardi \& Guimarães (2000).

As prevalências dos PGI foram calculadas de acordo com Bush et al. (1997). 
O escore corporal dos animais foi classificado como magro, bom e obeso (Greenacre 2003, Haire 2003). Além disto, a apresentação de sinais clínicos como apatia, perda de peso, diarreia, vômito, entre outros, foi utilizada para a avaliação do estado de saúde dos animais estudados. As informações pertinentes ao manejo sanitário dos animais e das instalações foram prestadas pelos profissionais responsáveis e também levantadas a partir do acompanhamento diário da rotina do CETAS.

\section{RESULTADOS}

Em total, foram coletadas 223 amostras fecais, das quais $135(60,3 \%)$ eram de aves e 88 (39,5\%) de mamíferos. Em 91 (40,8\%) amostras foram encontrados ovos e/ ou oocistos de nematódeos e/ou protozoários, sendo 64 $(70,3 \%)$ de aves e 27 (29,7\%) de mamíferos. No Quadro 1 , são apresentadas as prevalências dos PGI por cada grupo de animais analisados. Eimeria sp. (89,1\%), Ascaridida $(67,2 \%)$, Capillaria sp. $(39,1 \%)$ e Spirurida $(15,6 \%)$ foram os PGI identificados nas amostras fecais das aves, enquanto Strongylida (92,6\%), Coccídios (74,1\%), Trichuris trichiura $(70,4 \%)$, Strongyloides sp. $(70,4 \%)$, Toxocara canis $(3,7 \%)$ e Ancylostoma sp. (3,7\%) foram os PGI identificados nos mamíferos. Aves da ordem Accipitriformes e mamíferos da família Cebidae apresentaram maior diversidade de PGI. Os
PGI identificados e seus respectivos hospedeiros são apresentados no Quadro 2.

Carrapatos, piolhos, pulgas e moscas hematófagas foram os ectoparasitos coletados nas aves e mamíferos estudados (Quadro 3). A diversidade de ectoparasitos foi maior nas aves. Os piolhos mastigadores identificados em aves

Quadro 1. Prevalência de parasitos gastrointestinais em aves e mamíferos do Centro de Triagem de Animais Silvestres (CETAS) do Estado de Pernambuco, setembro de 2012 a fevereiro de 2013

\begin{tabular}{lccc}
\hline \multicolumn{1}{c}{ Hospedeiros } & $\mathrm{N}$ & $\mathrm{P}$ & $\%$ \\
\hline Aves, Psittaciformes & 95 & 42 & 44,2 \\
Aves, Accipitriformes & 19 & 18 & 94,7 \\
Aves, Falconiformes & 11 & 04 & 36,4 \\
Aves, Strigiformes & 06 & 0 & 0,0 \\
Aves, Piciformes & 03 & 0 & 0,0 \\
Aves, Gruiformes & 01 & 0 & 0,0 \\
Mammalia, Primates & 78 & 25 & 32,1 \\
Mammalia, Carnivora & 08 & 02 & 25,0 \\
Mammalia, Rodentia & 01 & 0 & 0,0 \\
Mammalia, Xenarthra & 01 & 0 & 0,0 \\
Total & 223 & 91 & 40,8
\end{tabular}

$\mathrm{N}$ = número de amostras coletadas; $\mathrm{P}=$ número de amostras com parasitos gastrointestinais; \% = prevalência.

Quadro 2. Parasitos gastrointestinais detectados em amostras fecais de aves e mamíferos do Centro de Triagem de Animais Silvestres (CETAS) do Estado de Pernambuco, setembro de 2012 a fevereiro de 2013

\begin{tabular}{|c|c|c|c|c|}
\hline Hospedeiros & Espécies estudadas & $\mathrm{N}$ & $\mathrm{P}$ & Parasitos gastrointestinais \\
\hline \multirow[t]{2}{*}{ Aves, Accipitriformes } & Rupornis magnirostris Gmelin,1788 (Gavião-carijó) & 11 & 10 & Capillaria sp., Spirurida e Eimeria sp. \\
\hline & Parabuteo unicinctus Temminck, 1824 (Gavião-asa-de-telha) & 08 & 08 & Capillaria sp., Ascaridida e Eimeria sp. \\
\hline \multirow[t]{3}{*}{ Aves, Falconiformes } & Falco sparverius Linnaeus, 1758 (Quiriquiri) & 01 & 0 & - \\
\hline & Caracara plancus Miller, 1777 (Caracará) & 08 & 03 & Eimeria sp. \\
\hline & Mivalgo chimachima Vieillot, 1816 (Carrapateiro) & 02 & 01 & Eimeria sp. \\
\hline \multirow[t]{3}{*}{ Aves, Strigiformes } & Tyto alba Scopoli, 1769 (Coruja-da-igreja) & 02 & 0 & - \\
\hline & Megascops choliba Vieillot, 1817 (Corujinha-do-mato) & 03 & 0 & - \\
\hline & Asio clamator Vieillot, 1808 (Coruja-orelhuda) & 01 & 0 & - \\
\hline \multirow[t]{12}{*}{ Aves, Psittaciformes } & Ara ararauna Linnaeus, 1758 (Arara-canindé) & 08 & 03 & Capillaria sp. \\
\hline & Ara macao Linnaeus, 1758 (Araracanga) & 03 & 02 & Capillaria sp. \\
\hline & Ara chloropterus Gray, 1859 (Arara-vermelha-grande) & 02 & 01 & Capillaria sp. \\
\hline & Anodorhynchus hyacinthinus Latham, 1790 (Arara-azul-grande) & 01 & 01 & Capillaria sp. \\
\hline & Amazona aestiva Linnaeus, 1758 (Papagaio-verdadeiro) & 54 & 35 & Ascaridida e Eimeria sp. \\
\hline & Amazona amazonica Linnaeus, 1766 (Curica) & 09 & 0 & - \\
\hline & Amazona farinosa Boddaert, 1783 (Papagaio-moleiro) & 01 & 0 & - \\
\hline & Amazona vinacea Kuhl, 1820 (Papagaio-de-peito-roxo) & 01 & 0 & - \\
\hline & Guarouba guarouba Gmelin, 1788 (Ararajuba) & 01 & 0 & - \\
\hline & Primolius auricollis Cassin, 1853 (Maracanã-de-colar) & 01 & 0 & - \\
\hline & Pionus fuscus Statius Muller, 1776 (Maitaca-roxa) & 02 & 0 & - \\
\hline & Aratinga leucophthalma Statius Muller, 1776 (Periquitão-maracanã) & 02 & 0 & - \\
\hline \multirow[t]{2}{*}{ Aves, Psittaciformes } & Aratinga cactorum Kuhl, 1820 (Periquito-da-caatinga) & 09 & 0 & - \\
\hline & Brotogeris chiriri Vieillot, 1818 (Periquito-de-encontro-amarelo) & 01 & 0 & - \\
\hline \multirow[t]{2}{*}{ Aves, Piciformes } & Ramphastos toco Statius Muller, 1776 (Tucanuçu) & 01 & 0 & - \\
\hline & Ramphastos tucanus Linnaeus, 1758 (Tucano-grande-de-papo-branco) & 02 & 0 & - \\
\hline Aves, Gruiformes & Psophia viridis Spix, 1825 (Jacamim-de-costas-verdes) & 01 & 0 & - \\
\hline \multirow[t]{2}{*}{ Primates, Cebidae } & Sapajus libidinosus Spix, 1823 (Macaco-prego) & 17 & 16 & $\begin{array}{l}\text { Strongylida, Strongyloides sp., } \\
\text { Trichuris trichiura e Coccídios }\end{array}$ \\
\hline & Sapajus flavius Schreber, 1774 (Macaco-prego-galego) & 03 & 03 & $\begin{array}{l}\text { Strongylida, Strongyloides sp., } \\
\text { Trichuris trichiura e Coccídios }\end{array}$ \\
\hline \multirow[t]{2}{*}{ Primates, Callitrichidae } & Callithrix jacchus Linnaeus, 1758 (Sagui-de-tufos-brancos) & 55 & 06 & Strongylida \\
\hline & Callithrix penicilata É. Geoffroy, 1812 (Sagui-de-tufos-pretos) & 03 & 0 & - \\
\hline Carnivora, Procyonidae & Nasua nasua Linnaeus, 1766 (Quati) & 03 & 01 & Coccídios \\
\hline Carnivora, Canidae & Cerdocyon thous Linnaeus, 1766 (Cachorro-do-mato) & 04 & 01 & Toxocara canis e Ancylostoma sp. \\
\hline Carnivora, Mustelidae & Eira barbara Linnaeus, 1758 (Papa-mel) & 01 & 0 & - \\
\hline Rodentia, Cuniculidae & Cuniculus paca Linnaeus, 1766 (Paca) & 01 & 0 & - \\
\hline Xenarthra, Bradypodidae & Bradypus variegatus Schinz, 1825 (Preguiça-de-três-dedos) & 01 & 0 & - \\
\hline
\end{tabular}

$\mathrm{N}$ = número de espécimes examinados; $\mathrm{P}$ = número de espécimes com parasitos gastrointestinais. 
Quadro 3. Ectoparasitos de aves e mamíferos do Centro de Triagem de Animais Silvestres (CETAS) do Estado de Pernambuco, setembro de 2012 a fevereiro de 2013

\begin{tabular}{|c|c|c|}
\hline Hospedeiros & Espécies estudadas & Ectoparasitos \\
\hline Aves, Accipitriformes & Rupornis magnirostris Gmelin,1788 (Gavião-carijó) & $\begin{array}{c}\text { Colpocephalum turbinatum e Kurodaia } \\
\text { (Kurodaia) fulvofasciata }\end{array}$ \\
\hline Aves, Procellariiformes & Calonectris borealis Cory, 1881 (Bobo-grande) & $\begin{array}{c}\text { Halipeurus sp., Naubates sp., Saemundssonia sp. } \\
\text { e Austromenopon sp. }\end{array}$ \\
\hline \multirow[t]{2}{*}{ Aves, Psittaciformes } & Ara chloropterus Gray, 1859 (Arara-vermelha-grande) & Paragoniocotes sp. \\
\hline & Amazona aestiva Linnaeus, 1758 (Papagaio-verdadeiro) & Brueelia sp. \\
\hline Aves, Piciformes & Ramphastos toco Statius Muller, 1776 (Tucanuçu) & Myrsidea sp. \\
\hline Aves, Pelecaniformes & Ardea alba Linnaeus, 1758 (Garça-branca-grande) & Pseudolynchia sp. \\
\hline Carnivora, Procyonidae & Nasua nasua Linnaeus, 1766 (Quati) & Rhipicephalus sanguineus \\
\hline Xenarthra, Bradypodidae & Bradypus variegatus Schinz, 1825 (Preguiça-de-três-dedos) & Amblyomma varium \\
\hline Xenarthra, Myrmecophagidae & Tamandua tetradactyla Linnaeus, 1758 (Tamanduá-mirim) & $\begin{array}{c}\text { Amblyomma calcaratum, } \text { A. nodosum e } \\
\text { Ornithodoros talaje }\end{array}$ \\
\hline Carnivora, Canidae & Cerdocyon thous Linnaeus, 1766 (Cachorro-do-mato) & Rhipicephalus sanguineus e Ctenocephalides felis fel \\
\hline
\end{tabular}

foram: Colpocephalum turbinatum, Kurodaia (Kurodaia) fulvofasciata, Austromenopon sp., Myrsidea sp. (Amblycera, Menoponidae); Halipeurus sp., Naubates sp., Saemundssonia sp., Paragoniocotes sp. e Brueelia sp. (Ischnocera, Philopteridae) (Quadro 3). Moscas hematófagas da espécie Pseudolynchia sp. foram coletadas unicamente em Ardea alba (garça-branca-grande) (Quadro 3).

Nos mamíferos, os ectoparasitos identificados foram os carrapatos Rhipicephalus sanguineus, Amblyomma varium, A. calcaratum, A. nodosum (Acari, Ixodidae) e Ornithodorostalaje (Acari, Argasidae), além da pulga Ctenocephalides felis felis (Siphonaptera, Pulicidae).

A maioria dos animais apresentava boa condição corporal e nenhum dos animais parasitados apresentou sinais clínicos associados com a infecção e/ou infestação. No entanto, as condições estruturais e sanitárias do CETAS-PE foram avaliadas como deficientes e precárias, guardando relação direta com os parasitos identificados.

\section{DISCUSSÃO}

A prevalência de parasitos relatada no presente estudo é considerada como uma ameaça à saúde dos animais (Daszak et al. 2000), uma vez que os PGI e os ectoparasitos podem atuar como oportunistas ou como agentes primários de doença (Freitas et al. 2001 e 2002a, Catão-Dias 2003, Godoy \& Cubas 2011, Oliveira et al. 2011, Santos et al. 2011). A maioria dos PGI identificados em aves e mamíferos são monoxenos, os quais são mais frequentes devido à maior facilidade para infectar seus hospedeiros (Freitas et al. 2002a, Urquhart et al. 2008, Sibaja-Morales et al. 2009, Santos et al. 2011), enquanto os parasitos heteroxenos são transmitidos por hospedeiros intermediários (normalmente invertebrados) (Urquhart et al. 2008). 0 cativeiro desfavorece a infecção por uma grande variedade de grupos/espécies de parasitos, uma vez que as fontes de infecção são limitadas, ao contrário do que ocorre em vida livre, onde as fontes de infecção são mais abundantes (Freitas et al. 2001, Sibaja-Morales et al. 2009, Santos et al. 2011). Apesar disto, aves da ordem Accipitriformes e mamíferos da família Cebidae apresentaram maior diversidade de PGI, talvez por terem ingressado parasitados no CETAS e não terem sido posteriormente tratados.

O maior número de amostras fecais de aves se deve ao fato de que, tanto em âmbito nacional como regional, as aves representam cerca de $82 \%$ dos animais mantidos em zoológicos e centros de triagem (Pagano et al. 2009). As aves silvestres albergam uma grande diversidade de parasitos, sendo mais frequentes os nematódeos dos gêneros Capillaria (ordem Enoplida), Ascaridia e Contracaecum (ordem Ascaridida), Physaloptera, Synhimantus, Tetrameres e Procyrnea (ordem Spirurida), além dos protozoários do grupo dos Coccídios (gêneros Eimeria e Isospora) (Vicente et al. 1995, Freitas et al. 2002a, Silva et al. 2009, Santos et al. 2011, Melo et al. 2012 e 2013). No presente estudo, Capillaria sp., Eimeria sp. e nematódeos das ordens Ascaridida e Spirurida foram detectados unicamente nas fezes de Psittaciformes (Ara ararauna, A. macao, A. chloropterus, Anodorhynchus hyacintinus e Amazona aestiva), Accipitriformes (Rupornis magnirostris e Parabuteo unicinctus) e Falconiformes (Caracara plancus e Mivalgo chimachima), o que também foi registrado por Freitas et al. (2002a) em aves em cativeiro no Parque Estadual Dois Irmãos em Recife, Pernambuco, a partir da análise de amostras fecais. No CETAS de João Pessoa, Paraíba, Melo et al. (2013) descreveram, através de necropsias, o parasitismo pelos nematódeos Ascaridia hermaphrodita e Synhimantus (Synhimantus) rectus em $A$. aestiva e $R$. magnirostris, respectivamente. Em aves Strigiformes em cativeiro em Santa Maria, Rio Grande do Sul, Silva et al. (2009) registraram a ocorrência de Capillaria sp., Strongylida e Coccídios em amostras fecais, o que difere do presente estudo, onde não foram detectados parasitos nas amostras das aves deste grupo. As técnicas diagnósticas (parasitológico de fezes ou necropsia) e a área geográfica explicam as diferenças encontradas nos mencionados estudos.

Os PGI identificados em mamíferos no presente estudo foram também registrados por Freitas et al. (2001) no Parque Estadual Dois Irmãos, Pernambuco. No presente estudo, os primatas não humanos apresentaram uma maior diversidade de PGI (Strongyloides sp., Strongylida, T. trichiura e Coccídios). Estes animais são hospedeiros de duas espécies de Strongyloides: S. stercoralis e S. fuellebor$n i$, ambas com potencial zoonótico. Na ordem Strongylida, o parasitismo por nematódeos dos gêneros Ancylostoma, Molineus, Oesophagostomum e Trichostrongylus foi relatado em primatas não humanos em cativeiro e em vida livre (Vicente et al. 1995, Legesse \& Erko 2004, Mbaya \& Udendeye 2011, Kouassi et al. 2015); T. trichiura (ordem 
Enoplida) também foi diagnosticado neste grupo animais (Mbaya \& Udendeye 2011). Nematódeos do gênero Trichuris são um dos PGI de maior dificuldade para o controle, o que se deve basicamente a dois fatores: (1) os antihelmínticos são muito eficazes contra os parasitos adultos, mas não contra as larvas, que penetram profundamente na mucosa do ceco, dificultando a exposição ao fármaco; (2) os ovos são muito resistentes às condições ambientais e podem sobreviver por longos períodos, o que favorece a ocorrência de reinfecções constantes (Lindsay \& Blagburn 1995, Urquhart et al. 2008). É importante destacar que, assim como os ovos de T. trichiura, os ovos de Capillaria sp., T. canis, os dos Ascarídeos de aves e os oocistos de Coccídios, são bastante resistentes a agentes físicos e químicos, dificultando o controle ambiental (Lindsay \& Blagburn 1995, Freitas et al. 2001 e 2002a, Urquhart et al. 2008, Sibaja-Morales et al. 2009, Santos et al. 2011). Em cativeiro, os animais vivem continuamente em gaiolas e/ou recintos contaminados, o que facilita a reinfecção, mesmo que o tratamento antiparasitário seja realizado periodicamente (Freitas et al. 2002a, Santos et al. 2011). No CETAS-PE, os antiparasitários são utilizados unicamente quando os animais manifestam sinais clínicos como apatia, diarreia e perda de peso, o que favorece a manutenção dos parasitos nos animais e no ambiente. Os sinais clínicos relacionados com os PGI são inespecíficos e a maioria das infecções parasitárias é assintomática (Deem et al. 2008, Urquhart et al. 2008, Godoy \& Cubas 2011). Portanto, não é recomendável vermifugar os animais unicamente mediante a apresentação de sinais clínicos (Freitas et al. 2001 e 2002a, Deem et al. 2008, Sibaja-Morales et al. 2009, Santos et al. 2011).

Mesmo parasitados por PGI considerados como muito patogênicos, como Eimeria sp., T. trichiura, T. canis e Ancylostoma sp., as aves e mamíferos estavam em boa condição física e não apresentavam sinais clínicos associados com a infecção parasitária, o que foi também registrado em outros estudos (Freitas et al. 2001 e 2002a, Sibaja-Morales et al. 2009, Silva et al. 2009, Santos et al. 2011). No entanto, a presença destes PGI deve ser encarada como uma ameaça à saúde dos animais, pois a maioria dos parasitos comporta-se como oportunista, mas em situações de imunocomprometimento, frequentes em ambientes de cativeiro, os animais parasitados podem manifestar sinais clínicos (Freitas et al. 2001 e 2002a, Catão-Dias 2003, Deem et al. 2008, Godoy \& Cubas 2011, Santos et al. 2011). Diarreia, emagrecimento, apatia e morte podem ocorrer quando a carga parasitária é elevada (Freitas et al. 2001 e 2002a, Deem et al. 2008, Sibaja-Morales et al. 2009, Santos et al. 2011). A obstrução intestinal provocada pelo nematódeo Ascaridia hermaphrodita foi responsável pela morte de 14 psitacídeos no CETAS de João Pessoa-PB (Melo et al. 2013). Fizeram parte deste estudo, três espécimes de Sapajus flavius (Macaco-prego-galego), os quais estavam parasitados por Strongylida, Strongyloides sp., T. trichiura e Coccídios. Este dado reveste-se de importância uma vez que $S$. flavius se encontra criticamente ameaçado de extinção (IUCN 2011). Amostras fecais de outras espécies ameaçadas, a exemplo de Amazona vinacea (Papagaio-de-peito-roxo) e Guarouba guarouba (Ararajuba) (UICN 2011), também foram analisadas, mas não foram encontrados PGI nestas aves. 0 conhecimento sobre a fauna parasitária de animais selvagens é fundamental para os estudos de impactos na saúde, principalmente de espécies ameaçadas (Muniz-Pereira et al. 2009). Neste sentido, o diagnóstico coproparasitológico e o controle de infecções parasitárias devem ser parte da rotina de protocolos sanitários em animais de cativeiro (UICN 1998, Felasa 1999, Freitas et al. 2001, 2002a, Deem et al. 2008, Sibaja-Morales et al. 2009, Santos et al. 2011, Melo et al. 2013). A realização periódica de exames coproparasitológicos é fundamental para orientar médicos veterinários e biólogos sobre a necessidade ou não da utilização de produtos antiparasitários, o que evitará gastos desnecessários e diminuirá também o risco de mortalidade (Deem et al. 2008, Sibaja-Morales et al. 2009, Oliveira et al. 2011, Santos et al. 2011).

Os piolhos mastigadores (ordem Mallophaga) foram os ectoparasitos mais frequentes nas aves estudadas, o que difere do estudo realizado por Freitas et al. (2002b) em aves mantidas em cativeiro no Parque Dois Irmãos e em um criatório científico em Recife-PE, onde os ácaros plumícolas foram os ectoparasitos mais frequentes. A pediculose resulta, com frequência, da superlotação nas gaiolas e recintos, mesmo que os piolhos sejam ectoparasitos hospedeiro-específicos (Morishita et al. 2001, Freitas et al. 2002b, Urquhart et al. 2008, Oliveira et al. 2011). Embora nenhuma das aves infestadas apresentasse sintomatologia associada aos ectoparasitos, o conhecimento sobre a infestação por piolhos mastigadores em aves reveste-se de importância, uma vez que a pediculose pode causar transtornos como prurido, inquietação, perda do apetite, além de danos às penas e à pele (Freitas et al. 2002b, Oliveira et al. 2011). Por isso, medidas de caráter preventivo devem ser instituídas para minimizar os efeitos deletérios do ectoparasitismo (Morishita et al. 2001, Oliveira et al. 2011).

Moscas do gênero Pseudolynchia (Diptera, Hippoboscidae) parasitam vários grupos de aves e se destacam por serem hematófagas e atuarem como vetores de hemoparasitos do gênero Haemoproteus (Santos et al. 2011), que afetam a saúde das aves parasitadas (Atkinson et al. 2008).

De maneira similar ao registrado neste estudo, o carrapato $R$. sanguineus foi também descrito em Nasua nasua (Quati) no Parque Dois Irmãos e no Parque Zoobotânico Arruda Câmara em João Pessoa-Paraíba (Dantas-Torres et al. 2010), enquanto Galactis cuja (Furão) foi registrado como hospedeiro deste carrapato no CETAS de São Luís-MA (Figueiredo et al. 2010). Esta é a espécie de carrapato de mais ampla distribuição geográfica no mundo, sendo conhecida popularmente como "carrapato-vermelho-do-cão" (Barros-Battesti et al. 2006, Dantas-Torres et al. 2010). É um ectoparasito trioxeno que realiza todas as mudas antes da troca de hospedeiros no ambiente (Urquhart et al. 2008). Carnívoros silvestres, em cativeiro ou que vivem em áreas frequentadas por cães, atuam como hospedeiros deste carrapato (Barros-Battesti et al. 2006). 0 parasitismo misto por A. nodosum e A. calcaratum em Tamandua tetradactyla (Tamanduá-mirim), conforme relatado no presente estudo, é considerado frequente (Barros-Battesti et al. 2006). Dantas-Torres et al. (2010) registraram o parasitis- 
mo por A. nodosum em Myrmecophaga tridactyla (Tamanduá-bandeira) e T. tetradactyla no Parque Dois Irmãos em Pernambuco e no Parque Zoobotânico Arruda Câmara da Paraíba. No CETAS do Maranhão, Figueiredo et al. (2010), reportaram o parasitismo por Amblyomma sp. em T. tetradactyla. Os carrapatos do gênero Amblyomma são também trioxenos (realizam no ambiente todas as mudas para troca de hospedeiros) e as espécies $A$. nodosum e $A$. calcaratum parasitam, quase que exclusivamente, tamanduás na fase adulta e aves na fase imatura (larvas e ninfas) (Barros-Battesti et al. 2006, Urquhart et al. 2008).

Assim como no presente estudo, o carrapato $A$. varium foi descrito em Bradypus variegatus (Preguiça-de-três-dedos) no Parque Zoobotânico Arruda Câmara em João Pessoa (Dantas-Torres et al. 2010). Este carrapato é considerado, na fase adulta, como específico de preguiças.

Segundo Barros-Battesti et al. (2006), o carrapato $O$. talaje parasita várias espécies de mamíferos. Este é o primeiro registro de 0 . talaje em Pernambuco e T. tetradactyla é apresentado como novo hospedeiro deste argasídeo, que passa a maior parte do seu ciclo biológico no ambiente.

A pulga Ctenocephalides felis felis foi identificada unicamente em Cerdocyon thous (Cachorro-do-mato), enquanto que no CETAS do Maranhão, o parasitismo por esta pulga foi descrito unicamente em carnívoros da espécie $N$. nasua (Figueiredo et al. 2010).

É importante destacar que $95 \%$ da população de carrapatos e pulgas se desenvolve no ambiente, sendo de fundamental importância o controle ambiental destes ectoparasitos para evitar a propagação dos mesmos para outros animais, pois estes artrópodes se destacam por sua baixa especificidade de hospedeiros (Urquhart et al. 2008).

As precárias condições estruturais e sanitárias do CETAS-PE guardam relação direta com os parasitos identificados neste estudo e devem ser levadas em consideração para a adoção de medidas adequadas de controle. Desafortunadamente, as instalações do CETAS-PE não são adequadas para receber a grande quantidade de animais que, diariamente, são recebidos nesta instituição, o que tem resultado em superlotação das gaiolas e/ou recintos. Em condições de superlotação, a proximidade entre os animais favorece a transmissão tanto de PGI quanto de ectoparasitos. Além disto, devido ao grande fluxo de animais apreendidos do tráfico, algumas vezes, os animais albergados há mais tempo são retirados das gaiolas e/ou recintos e transferidos pra outros locais, para dar lugar aos animais de ingresso recente, favorecendo a transmissão de PGI monoxenos (os mais prevalentes neste estudo). Por questões de logística, a higienização das gaiolas e/ou recintos se restringe unicamente à remoção dos resíduos gerados pelos animais (fezes e restos de alimentos). Evidentemente, este manejo sanitário deficiente favorece a manutenção dos parasitos no ambiente, principalmente daqueles mais resistentes. Neste sentido, a limpeza e desinfecção sistemática de gaiolas e/ou recintos deve ser priorizada, utilizando principalmente a vassoura de fogo para a destruição de ovos e oocistos de PGI, além da remoção e troca do substrato do piso (Sibaja-Morales et al. 2009). Além destas medidas, a utilização de acaricidas/inseticidas no ambien- te é fundamental para o controle das fases ambientais dos carrapatos e das pulgas.

A partir do diagnóstico dos parasitos apresentados, pretende-se contribuir não só com a saúde dos animais silvestres mantidos no CETAS-PE, mas também com a saúde das pessoas envolvidas diretamente com o manejo dos mesmos e com a saúde ambiental. Neste sentido, o diagnóstico de parasitos com potencial zoonótico tais como T. trichiura, Strongyloides sp., T. canis e Ancylostoma sp., merece destaque não somente porque são um risco para a saúde dos animais, mas também porque podem afetar severamente a saúde das pessoas que trabalham diretamente com estes animais, tais como veterinários, biólogos, zootecnistas, tratadores, entre outros (Freitas et al. 2001, Sibaja-Morales et al. 2009, Public Health Agency of Canada, 2011). Strongyloides fuelleborni e S. stercoralis também apresentam potencial zoonótico, já que são descritos em humanos e primatas não humanos (Mbaya \& Udendeye 2011, Viney \& Lok 2011, Mafuyai et al. 2013, Kouassi et al. 2015). Infestações por carrapatos dos gêneros Amblyomma e Ornithodoros (Barros-Battesti et al. 2006, Dantas-Torres et al. 2010) são frequentes em humanos, provocando reações que variam desde prurido e lesões na pele até distúrbios sistêmicos (Reck et al. 2014). Pouco se conhece sobre o risco de transmissão de doenças por estes carrapatos em Pernambuco (Dantas-Torres et al. 2010). Estes resultados pressupõem a adoção de medidas de biossegurança para diminuir o risco zoonótico representado pelos PGI e ectoparasitos diagnosticados.

Os resultados obtidos aportam informações relevantes sobre a fauna parasitária de aves e mamíferos em cativeiro no Brasil. Os carrapatos A. calcaratum e O. talaje são registrados pela primeira vez em Pernambuco e T. tetradactyla é apresentado como novo hospedeiro de 0 . talaje. A elevada prevalência de PGI é uma ameaça à saúde dos animais e das pessoas envolvidas no cuidado diário com os mesmos, uma vez que foram diagnosticados tanto PGI muito patogênicos para os animais como com potencial zoonótico. As precárias condições estruturais e sanitárias do CETAS-PE estão relacionadas com os parasitos identificados neste estudo e devem ser levadas em consideração para a adoção de medidas adequadas de controle. Os resultados deste estudo contribuirão de maneira significativa para a conservação de animais selvagens no CETAS-PE e para a saúde dos profissionais responsáveis pela manutenção destes animais.

Agradecimentos.- Ao CETAS-PE por fornecer a licença para a realização das coletas e a todos os funcionários deste órgão pelo apoio técnico e logístico.

\section{REFERÊNCIAS}

Aragão H. \& Fonseca F. 1961. Notas de Ixodologia. VIII. Lista e chave para os representantes da fauna ixodológica brasileira. Mem. Inst. Oswaldo Cruz 59:131-148.

Atkinson C.T., Thomas N.J. \& Hunter D.B. 2008. Parasitic diseases of wild birds. Blackwell, Iowa. 310p.

Barros-Battesti D.M., Arzua M. \& Bechara G.H. 2006. Carrapatos de Importância Médico-Veterinária da Região Neotropical: um guia ilustrado para identificação de espécies. Vox/ICTTD-3/Butantan, São Paulo. 223p.

Brasil - Instituto Brasileiro do Meio Ambiente e dos Recursos Naturais Re- 
nováveis 2003. Instrução Normativa no 3 de 27 de maio de 2003. Lista das Espécies da Fauna Brasileira Ameaçada de Extinção. IBAMA, Brasília. Disponível em <http://www.ibama.org.br> Acesso em 28 mar. 2012.

Bush A.O., Lafferty K.D., Lotz J.M. \& Shostak A.W. 1997. Parasitology meets ecology in its own terms: Margolis et al. revisited. J. Parasitol. 83:575583.

Catão-Dias J.L. 2003. Doenças e seus impactos sobre a biodiversidade. Ciência e Cultura 55:32-34.

Dantas-Torres F., Ferreira D.R., Melo L.M., Lima P.A., Siqueira D.B., Rameh-de-Albuquerque L.C., Melo A.V. \& Ramos J.A., 2010. Ticks on captive and free-living wild animals in northeastern Brazil. Exp. Appl. Acarol. 50:181-189.

Daszak P., Cunningham A.A. \& Hyatt A.D. 2000. Emerging infectious diseases of wildlife: threats to biodiversity and human health. Science 87:443449.

Deem S.L., Ladwing E., Cray C., Karesh W.B. \& Amato G. 2008. Health assessment of the ex situ population of St Vicent parrots (Amazona guildingii) in St Vicent and the Grenadines. J. Avian Med. Surg. 22:114-122.

Felasa - Federation for Laboratory Animal Science Associations 1999. Health monitoring of non-human primate colonies. Laboratory Animals 33(Suppl.1):S1-S16.

Figueiredo M.A.P., Santos A.C.G. \& Guerra R.M.S.N.C. 2010. Ectoparasitos de animais silvestres no Maranhão. Pesq. Vet. Bras. 30:988-990.

Freitas M.F.L., Oliveira J.B., Cavalcanti M.D.B., Oliveira R.A. \& Evencio-Sobrinho A. 2001. Perfil coproparasitológico de mamíferos silvestres en cautiverio en el Estado de Pernambuco, Brasil. Parasitología al Día 25:121-125.

Freitas M.F.L., Oliveira J.B., Cavalcanti M.D.B., Leite A.S., Magalhães V.S., Oliveira R.A. \& Evencio-Sobrinho A. 2002a. Parásitos gastrointestinales aves silvestres en cautiverio en el estado de Pernambuco, Brasil. Parasitología al Día 57:50-54.

Freitas M.F.L., Botêlho M.C.N., Leite A.S., Magalhães V.S., Evencio-Sobrinho A., Oliveira R.A., Oliveira M.H.C. \& Oliveira J.B. 2002b. Ectoparasitos de aves silvestres mantidas em cativeiro no estado de Pernambuco, Brasil. Entomología y Vectores 9:25-33.

Godoy S.N. \& Cubas Z.S. 2011. Doenças virais e parasitárias em Psittaciformes: uma revisão. Clín. Vet. 90:32-44.

Greenacre C. 2003. The avian patient, p.5-29. In: Ballard B. \& Cheek R. (Eds), Exotic Animal Medicine for the Veterinary Technician. Blackwell, USA. $457 \mathrm{p}$.

Haire M. 2003. The role of the veterinary technician in wildlife rehabilitation, p.237-306. In: Ballard B. \& Cheek R. (Eds), Exotic Animal Medicine for the Veterinary Technician. Blackwell, USA. 457p.

Kouassi R.Y.W., McGraw S.W., Yao P.K., Abou-Bacar A., Brunet J., Pesson B., Bonfoh B., N'goran E.K. \& Candolfi E. 2015. Diversity and prevalence of gastrointestinal parasites in seven non-human primates of the Taï National Park, Côte d'Ivoire. Parasite 22:1-12.

Legesse M. \& Erko B. 2004. Zoonotic intestinal parasites in Papio anubis (baboon) and Cercopithecus aethiops (vervet) from four localities in Ethiopia. Acta Tropica 90:231-239.

Linardi P.M. \& Guimarães L.R. 2000. Sifonápteros do Brasil. Museu de Zoologia, USP/Fapesp, São Paulo, 291p.

Lindsay D.S. \& Blagburn B.L. 1995. Practical treatment and control of infections caused by canine gastrointestinal parasites. Vet. Med. 90:441455.

Mafuyai H.B., Barshep Y., Audu B.S., Kumbak D. \& Ojobe T.0. 2013. Baboons as potential reservoirs of zoonotic gastrointestinal parasite infections at Yankari National Park, Nigeria. Afr. Health Sci. 13:252-254.

Martins T.F., Onofrio V.C., Barros-Battesti D.M. \& Labruna M.B. 2010. Nymphs of the genus Amblyomma (Acari: Ixodidae) of Brazil: descriptions, redescriptions, and identification key. Ticks Tick-borne Dis. 1:7599.

Mbaya A.W. \& Udendeye U.J. 2011. Gastrointestinal parasites of captive and free-roaming primates at the Afi Mountain Primate Conservation Area in Calabar, Nigeria and their zoonotic implications. Pak. J. Biol. Sci. 14:709-714.

Melo C.M.F., Oliveira J.B., Athayde A.C.R., Dantas A.F.M., Feitosa T.F., Vilela V.L.R., Menezes D.J.A. \& Wagner P.G.C. 2012. Identification of parasites in Puffinus puffinus (Birds, Procellariiformes) from Northeastern Brazil. Vet. Res. Commun. 36:235-238.

Melo C.M.F., Oliveira J.B., Feitosa T.F., Athayde A.C.R., Dantas A.F.M., Menezes D.J.A., Vilela V.L.R., Wagner P.G.C. \& Febrônio A.B. 2013. Parasites of Psittaciformes and Accipitriformes in Paraíba state, northeastern Brazil. Revta Bras. Parasitol. Vet. 22:314-317.

Morishita T.Y., Mertins J.W., Baker D.G., Monahan C.M. \& Brook D.L. 2001. Ocurrence and species of lice on free-living and captive raptors in California. J. Avian Med. Surg. 15:288-292.

Muniz-Pereira L.C., Vieira F.M. \& Luque J.L. 2009. Checklist of helminth parasites of threatened vertebrate species from Brazil. Zootaxa 2123:1-45.

Oliveira J.B., Santos T., Vaughan C. \& Santiago H. 2011. External parasites of raptors (Falconiformes and Strigiformes): identification in an ex situ population from Mexico. Rev. Biol. Trop. 59:1257-1264.

Pagano I.S.A., Sousa A.E.B.A., Wagner P.G.C. \& Ramos R.T.C. 2009. Aves depositadas no Centro de Triagem de Animais Silvestres do IBAMA na Paraíba: uma amostra do tráfico de aves silvestres no estado. Ornithologia 3:132-144.

Price R.D., Hellenthal R.A. \& Palma R.L. 2003. World checklist of chewing lice with host associations and keys to families and genera, p.1-488. In: Price R.D., Hellenthal R.A., Palma R.L., Johnson K.P. \& Clayton D.H. (Eds), The Chewing Lice: World checklist and biological overview. Natural History Survey Special Publication, Illinois, 24. 501p.

Public Health Agency of Canada 2011. Trichuris trichiura: pathogen safety data sheet - infectious substances. Disponível em <http://www.phac-aspc.gc.ca/lab-bio/res/psds-ftss/trichuris-trichiura-eng.php> Acesso em 7 Mai. 2015.

Reck J., Bandarra P., Pavarini S., Termignoni C., Driemeier D., Martins J.R. \& Guimarães J.A. 2014. Experimentally induced tick toxicosis in rats bitten by Ornithodoros brasiliensis (Chelicerata: Argasidae): a clinico-pathological characterization. Toxicon 88:99-106.

Santos T., Oliveira J.B., Vaughan C. \& Santiago H. 2011. Health of an ex situ population of raptors (Falconiformes and Strigiformes) in Mexico: diagnosis of internal parasites. Rev. Biol. Trop. 58:1265-1274.

Sibaja-Morales K.D., Oliveira J.B., Rocha A.E.J., Hernándes-Gamboa J., Prendas-Gamboa J., Arroyo-Murillo F., Sandí J., Nuñez Y. \& Baldi M. 2009. Gastrointestinal parasites and ectoparasites of Bradypus variegatus and Choloepus hoffmanni sloths in captivity from Costa Rica. J. Zoo Wildl. Med. 40:86-90.

Silva A.S., Zanette R.A., Lara V.M., Gressler L.T., Carregaro A.B., Santurio J.M. \& Monteiro S.G. 2009. Gastrointestinal parasites of owls (Strigiformes) kept in captivity in the Southern region of Brazil. Parasitol. Res. 104:485-487.

UICN 1998. Guías para reintroducciones de la UICN: preparada por el grupo de especialistas en reintroducción de la Comisión de Supervivencia de Especies de la UICN. International Union for Conservation of Nature, Reino Unido. Disponível em <https://portals.iucn.org/library/efiles/ documents/PP-005-Es.pdf> Acesso em 10 Abr. 2013.

IUCN 2011. Red List of Threatened Species. International Union for Conservation of Nature and Natural Resources, Reino Unido. Disponível em <http://www.iucnredlist.org/> Acesso em 26 Jul. 2014.

Urquhart G.M., Armour J., Duncan J.L., Dunn A.M. \& Jennings F.W. 2008. Veterinary Parasitology. 2nd ed. Blackwell, USA. 275p.

Vicente J.J., Rodrigues H.O., Gomes D.C. \& Pinto R.M. 1995. Nematóides do Brasil, IV: Nematóides de aves. Revta Bras. Zool. 12(S1):1-273.

Viney M.E. \& Lok J.B. 2007. Strongyloides spp. Disponível em <http://www. ncbi.nlm.nih.gov/pmc/articles/PMC3091011/> Acesso em 7 mai. 2015.

Zajac A.M. \& Conboy G.A. 2006. Veterinary Clinical Parasitology. 7th ed. Blackwell, USA. 102p. 